\title{
Pilot study outcomes from a resource-limited setting for a low-cost training program for laparoscopic surgical skills
}

\author{
Pamela Andreatta a,b,*, Joseph Perosky ${ }^{b}$, Jessica Klotz ${ }^{a}$, Charlotte Gamble ${ }^{b}$, Frank Ankobea ${ }^{c}$, \\ Kwabena Danso ${ }^{\mathrm{c}}$, Vanessa Dalton ${ }^{\mathrm{b}}$ \\ a Department of Urologic Surgery, University of Minnesota Medical School, Minneapolis, USA \\ ${ }^{\mathrm{b}}$ Department of Obstetrics and Gynecology, University of Michigan Medical School, Ann Arbor, USA \\ c Department of Obstetrics and Gynecology, Komfo Anokye Teaching Hospital, Kumasi, Ghana
}

\section{A R T I C L E I N F O}

\section{Article history:}

Received 29 August 2013

Received in revised form 23 October 2013

Accepted 2 February 2014

\section{Keywords:}

Laparoscopic surgery

Laparoscopic training

Low-cost training program

Medical education

Training initiatives for expanding the use of laparoscopic surgery in resource-limited regions could have significant benefits for the health outcomes and economies of the population [1].

The option to use laparoscopic surgical techniques depends on the availability of equipment, instruments, and trained providers. Even if a physical infrastructure supports laparoscopy, training surgeons to master the requisite techniques is resource intensive in terms of time, facilitation, and materials. Training challenges for the surgeon include learning to translate a 2D video display into a 3D mental model of the operative field, controlling the camera to optimize visualization, and manipulating the long-shafted instruments to account for fulcrum effects while maintaining surgical precision and efficiency.

Simulation-based training programs are frequently used to facilitate the acquisition of laparoscopic skills. These programs require substantial financial investment to establish and sustain-something few institutions can afford in resource-limited environments. Although some low-technology simulation solutions such as the Fundamentals of Laparoscopic Surgery have demonstrated efficacy in selected performance domains, their costs remain beyond the feasibility of most programs in resource-limited regions [2]. The aim of the present study was to evaluate the feasibility and baseline impact of a low-cost, low technology, and locally sourced simulation-based program for high-fidelity laparoscopic surgical training in a resource-limited environment.

\footnotetext{
* Corresponding author at: 420 Delaware Street S.E., A590-1 Mayo Memorial Building MMC 394, Minneapolis, MN 55455, USA. Tel.: + 1612626 4791; fax: + 16126263994. E-mail address: pandreat@umn.edu (P. Andreatta).
}

The study received an exemption after review by the institutional review boards at the University of Michigan and the Kwame-Nkrumah University of Science and Technology (KNUST). All participants provided informed consent prior to study participation. The sample comprised 18 faculty and house officers from KNUST and Komfo Anokye Teaching Hospital in Kumasi, Ghana. Four (22\%) participants were women and all participants had observed laparoscopy fewer than 2 times, with no procedural involvement.

Laparoscopic towers, equipment, and instruments were donated by the University of Michigan Medical School to help facilitate the development of laparoscopic surgery capabilities in Ghana. A comprehensive instructional program was developed (by P.A.) to include training exercises, performance criteria, objective feedback, and proficiency targets for learning novice-level laparoscopic surgical skills. The training exercises are outlined in Box 1. All training materials were selected based on cost sensitivity and local availability in order to encourage sustainability. Box trainers were built using wood, foam, heavy fabric, glue, and hardware hinges (Fig. 1). The course materials for the entire program cost less than US $\$ 30$ per participant. All materials were purchased and assembled in Ghana, with the exception of small foam pieces brought from the USA (later also locally sourced).

Each participant completed a 3-hour introductory session, which included hands-on familiarization with each of 7 exercises and training in laparoscopic surgical skills associated with tissue manipulation, not clinical or procedural knowledge. The exercises were designed with variable challenges, including the following: instrument control of escalating difficulty with variable complexity templates and differing size and shape foam pieces; cutting exercises using a long balloon and rubber bands; camera navigation through increasing complexity models; translocation along predefined paths; and dissection to focus on precision and tissue preservation.

Tissue damage is the primary cause of iatrogenic injury for surgical patients; therefore, tissue handling and associated damage to tissue were selected as the primary variable of interest for all exercises. Although time is associated with surgical expertise, it is not the primary indicator of surgical skill. Practice exercises were not time limited, unlike the time limits for the assessments, in order to provide participants the freedom to focus on development of tissue-handling skills with minimal damage. Participants completed training exercises individually, although peer-to-peer teaching was encouraged during practice sessions held daily over 12 consecutive days, following the initial session. A final 3- 
Box 1

The 7 program exercises, ordered from least challenging to most difficult.

Instrument control A

Objective: using laparoscopic graspers, manipulate and move 7.5-14.5-mm foam pieces with variable shapes to align within a specified template

- Template: 40 -piece grid $(8 \times 5)$

- Shapes: squares, circles, hearts, triangles (large and small), small stars

- Focus on accuracy, precision, and tissue damage

- Twenty-minute time limit

Instrument control B

Objective: using laparoscopic graspers, manipulate and move 7.5$14.5-\mathrm{mm}$ foam pieces with variable shapes to align within a specified template

- Template: 56-piece mandala

- Shapes: squares, circles, hearts, triangles (large and small), small stars

- Focus on accuracy, precision, and tissue damage

- Twenty-minute time limit

\section{Cutting}

Objective: using laparoscopic graspers and scissors, manipulate and cut 2 rubber bands at the specified demarcation

- Long balloon partially filled with water

- Dark mark on each band indicating cutting point

- Bands wrapped around balloon

- Marks wrapped within the deflated balloon tail

- Focus on accuracy, precision, and tissue damage

- Twenty-minute time limit

\section{Camera navigation}

Objective: manipulate a laparoscopic camera with 0 and 30 lenses following auditory navigation cues

- Eight abdominal models with numeric markers located through the cavity

- Models have increasing complexity

- Auditory cues prerecorded, administered through headphones

- Cues direct user how to progress from one marker to the next

- Focus on accuracy and precision

\section{Translocation}

Objective: using a laparoscopic grasper, move 10 cooked peas from one container to another by following a prescribed path

- Two models with predefined linear and curvilinear paths

- Placement cup has narrow hole through which the pea must be placed, undamaged

- Focus on accuracy, precision, and tissue damage

- Ten-minute time limit

\section{Dissection}

Objective: dissect a tangerine using any available laparoscopic instruments, as follows:

- Remove peel in as few pieces as possible
- Separate each segment from the others

- Remove all pith and seeds from each segment

- Focus on accuracy, precision, and tissue damage

- Ninety-minute time limit

\section{Instrument control C}

Objective: using laparoscopic graspers, manipulate, move, and remove thin adhesive backing from, and place 7.5-14.5-mm foam pieces with variable shapes to align within a specified template

- Template: 16-piece geometric design

- Shapes: squares, circles, hearts, triangles (large and small), small stars

- Focus on accuracy, precision, and tissue damage

- Twenty-minute time limit

hour immersive session enabled participants to perform each exercise, ensuring that everyone completed each exercise at least twice during the program. Proctors from KNUST and the University of Michigan were available to supervise and guide participants at the initial training, at each participant's first practice session, and at the final session.

Descriptive methods were used to evaluate the extent to which program activities and participant perceptions about the value of the training could be facilitated. To assess the ability of the local institution to sustainably facilitate the program, we tracked the number of participants who were able to complete the program in its entirety, and determined the availability and costs of required materials for maintaining the program. To assess the acceptability of the program to participants, a 6-point Likert scale was used to collect self-reported value ratings for each training exercise and the overall program for supporting the acquisition of laparoscopic skills (Supplementary Material S1). Descriptive methods were used to analyze the variables of interest, including mean \pm SD calculated with SPSS version 17 (IBM, Armonk, NY, USA).

The number of laparoscopic towers limited the maximum number of participants that could be accommodated over the 2-week program period to 20 , with 18 participants completing the training program in its entirety.

Participants rated instruction as valuable overall. Participants' ratings (mean \pm SD) were recorded for each exercise in the following areas: overall usefulness; use for skill development/maintenance; use for self-assessment; stimulates critical thinking; applies to real world; clinical operative reasoning; clinical judgment; dissection skills; instrument control; instrument familiarization; and operative clinical planning. Ratings for each exercise domain ranged from average to very good.

The pilot data indicate that a low-technology and low-cost training program for laparoscopic skills designed for sustainability using locally sourced materials is feasible to implement in resource-limited areas.

There are many benefits to using low-technology simulation, not least of which are the increased availability and accessibility of training associated with lower material costs. There is evidence that, compared with more expensive high-technology simulation, low-technology simulation can lead to equivalent task-related training outcomes and support objective performance assessment $[3,4]$. The present results indicate that, given the donation of functional laparoscopic equipment and instruments, this novel and low-cost simulation-based laparoscopic training program is feasible to implement and thought to be beneficial by surgeons in a limited-resource setting. We hope that this evidence will encourage others to donate refurbished or new equipment where it is needed, and for recipients to develop similar programs to ensure the sustainability of benefits that can arise from those contributed resources.

Supplementary data to this article can be found online at http://dx. doi.org/10.1016/j.ijgo.2013.10.030. 


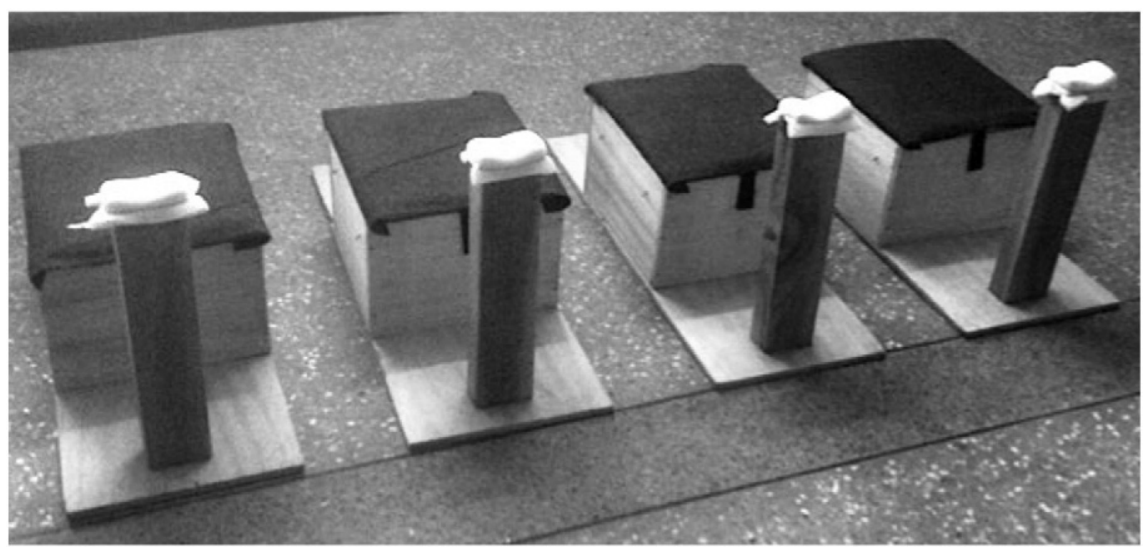

Fig. 1. Locally built laparoscopic box trainers.

\section{Acknowledgments}

The project was funded by a grant from the Center for Global Health at the University of Michigan.

\section{Conflict of interest}

The authors have no conflicts of interest.

\section{References}

[1] Bendinelli C, Leal T, Moncade F, Dieng M, Toure CT, Miccoli P. Endoscopic surgery in Senegal. Benefits, costs and limits. Surg Endosc 2002;16(10):1488-92.

[2] Okrainec A, Smith L, Azzie G. Surgical simulation in Africa: the feasibility and impact of a 3-day fundamentals of laparoscopic surgery course. Surg Endosc 2009;23(11):2493-8

[3] Diesen DL, Erhunmwunsee L, Bennett KM, Ben-David K, Yurcisin B, Ceppa EP, et al. Effectiveness of laparoscopic computer simulator versus usage of box trainer for endoscopic surgery training of novices. J Surg Educ 2011;68(4):282-9.

[4] Zheng B, Hur HC, Johnson S, Swanström LL. Validity of using Fundamentals of Laparoscopic Surgery (FLS) program to assess laparoscopic competence for gynecologists. Surg Endosc 2010;24(1):152-60. 